velocity hydrogen," Astron. Astrophys., vol. 7, pp. 381-404, Sept. 1970.

[35] F. J. Kerr and W. T. Sullivan, III, "The high-velocity hydrogen clouds considered as satellites of the galaxy," Astrophys. J., vol. 158, pp. 115-122, Oct. 1969.

[36] N. H. Dieter, "Berkeley survey of high-velocity interstellar neutral hydrogen: Discussion of results," A stron. Astrophys., vol. 12, pp. 59-75, Apr. 1971.

[37] G. L. Verschuur, "High-velocity clouds and 'normal' galactic structure," Astron. Astrophys., vol. 22, pp. 139-151, Jan. 1973.

[38] R. D. Davies, "Outer spiral structure and high velocity clouds in the galaxy," Nature, vol. 237, pp. 88-91, May 12, 1972.

[39] F. J. Kerr, "Properties of the neutral hydrogen in the Magellanic
Clouds," in The Magellanic Clouds, A. B. Muller, Ed. Dordrecht, The Netherlands: Reidel, 1971, pp. 50-65.

[40] M. S. Roberts, "The gaseous content of galaxies," in IAU Symp., no. 44 , pD. 12-36, 1972 .

[41] - "Integral properties of spiral and irregular galaxies," A stron. J., vol. 74, pp. 859-876, Sept. 1969.

[42] ——, "The detection of 21-centimeter hydrogen absorption arising from within the radio galaxy Centaurus A," Astrophys. J., vol. 161, pp. L9-L12, July 1970.

[43] - in press.

[44] J. B. Whiteoak and F. F. Gardner, "Neutral hydrogen absorption and emission in NGC 5128 (Centaurus A)," A strophys. Lett., vol. 8, pp. 57-60, Apr. 1971.

\title{
Introduction to Very-Long-Baseline Interferometry
}

\author{
MARSHALL H. COHEN
}

\begin{abstract}
Long-baseline interferometry achieves high angular resolution by using two or more widely separated radio telescopes and recording video signals on magnetic tapes, which are later brought together and crose-correlated. This paper contains discussions of the coherence and timing requirements and of calibration procedures. Applications to messuring brightness distributions and to spectroscopy are reviewed briefly. Some pertinent phenomena connected with radio-wave scattering in irregular media are discussed.
\end{abstract}

\section{INTRODUCTION}

I N THE EARLY and middle 1960's radio astronomers developed an appreciation of compact radio sources, usually associated with quasars or galactic nuclei. Their compactness is measured by angular size which is well under $1^{\prime}$ and in some cases less than $10^{-3 *}$. These sources are small but by no means weak, and their flux density is comparable to that of the extended sources, which may have $10^{10}$ times as much solid angle! Thus they are enormously brilliant and contain a very high energy density. They are mysterious fascinating objects and remain the subject of intense study.

A decade ago, angular size measurements were limited to about $0.1^{\prime}$, by the available techniques, and the much smaller size of the compact sources was being inferred on theoretical grounds. These grounds included the peaked nature of the spectrum and the variability of the sources, and were reasonably firm. Thus there was strong interest in making measurements at angular resolutions of $10^{-36}$ or better, to check the theoretical predictions and to see what the sources actually looked like on this scale.

Manuscript received February 26, 1973. Owens Valley Radio Observatory is supported by ONR under Contract N 00014-67-A00094-0019, and by NSF under Grants GP25225 and GP19400

The author is with the Owens Valley Radio Observatory and with the Department of Astronomy, California Institute of Technology, Pasadena, Calif. 91109.

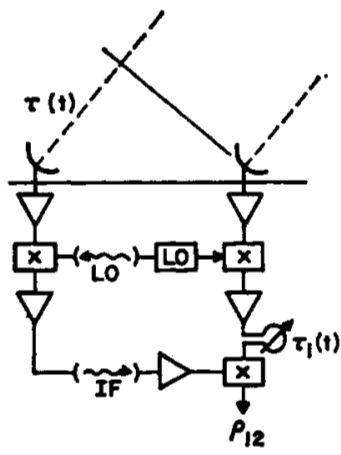

(a)

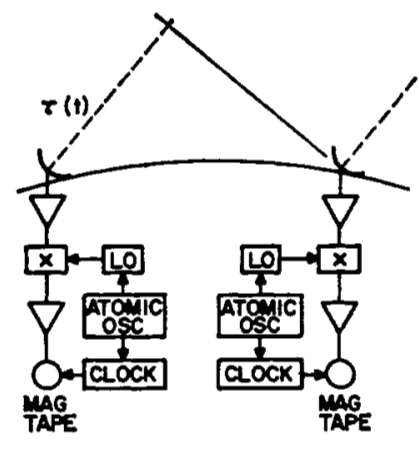

(b)
Fig. 1. (a) Conventional two-element interferometer with a radio link. (b) VLB system with independent atomic standard oscillators.

At that time high-resolution techniques included lunar occultations, interplanetary scintillations, and interferometry. The first two were limited by the scale of the diffraction patterns to about $0^{\prime \prime} .1$ but interferometry was limited only by practical considerations. Fig. 1(a) shows the conventional radio-link interferometer with the $\mathrm{LO}$ and IF signals transmitted by radio. In 1965 such interferometers were being used at baselines up to $120 \mathrm{~km}$. However, thousands of kilometers were really required, and it was decided to simplify the systems and eliminate the real-time links, as in Fig. 1(b). Two levelopments were necessary for this: a means of maintaining coherent independent local oscillators at the two telescopes, and a means of putting the video signals on magnetic tape for later processing. These developments were possible, and verylong-baseline (VLB) observations began in 1967. Baselines up to $10^{4} \mathrm{~km}$ and wavelengths down to $1.35 \mathrm{~cm}$ have now been used. However, the "later processing" has turned out to be an impediment to large-scale research, and the alternative of 
real-time cross correlation, via some appropriate link, is beginning to seem attractive.

The history and applications of VLB systems have been discussed in various early papers [1]-[8] and in several review articles [9]-[13]. Details of the National Radio Astronomy Observatory (NRAO) Mark II digital system are in the article by Clark [14] in this issue. Other pertinent articles in this issue include applications at meter wavelengths by Clark and Erickson [15], to continuum sources by Kellermann [16], to spectral-line sources by Welch [17], and to geodesy and fundamental astronomy by Counselman [18].

\section{INDEPENDENT LOCAL OSCILLATORS}

The oscillator stability required for VLB work depends on the nature of the experiments. There must at least be coherence for the minimum integration time set by the signal-tonoise ratio. But for some purposes-especially where fringe phases have to be compared-long-term stability is required. Atmospheric phase fluctuations are evidently involved in these matters also, but discussion of them is to be deferred until later. Atmospheric effects are important mainly at $\lambda>1 \mathrm{~m}$, while oscillator effects are important only at short wavelengths.

\section{A. Coherence}

The two oscillators will have a small frequency offset. This merely adds to the natural fringe rate (set by the earth's rotation) and is unimportant. What is important is the phase fluctuations. If $\phi$ is the phase difference between the two LO signals, then the coherence requirement is $\left\langle\phi^{2}\right\rangle_{\tau}^{1 / 2}<1$; i.e., the rms phase fluctuation must be less than 1 rad when averaged over the coherent integration time $\tau$, which usually is on the order of $1 \mathrm{~min}$. This requirement is now routinely met with atomic standard oscillators driving frequency synthesizers and multipliers.

Hydrogen maser oscillators generate the best sine waves. Several large observatories (Goldstone, Algonquin Park, Haystack, Green Bank) have these masers and their use has been very successful. At $13 \mathrm{~cm}$ they have allowed coherent integration for periods of up to a half-hour, with little or no loss in signal-to-noise ratio. Rubidium oscillators also have been used to generate LO signals at microwave frequencies. Their coherence time is on the order of $20 \mathrm{~s}$ at $3 \mathrm{~cm}$, but is many minutes at $6 \mathrm{~cm}$. A good crystal oscillator has smaller short-term fluctuations than the rubidium, but cannot be used at microwave frequencies because its frequency drifts rapidly (large long-term phase fluctuations). It is possible, however, to override the second characteristic by phase-locking a high-quality crystal oscillator to a rubidium oscillator with a time constant of about a minute. This gives a coherence time of $1 \mathrm{~min}$ at $\lambda=3 \mathrm{~cm}$. The superiority of this combination has been directly demonstrated in VLB observations between the NRAO in Green Bank, W. Va., and the Owens Valley Radio Observatory (OVRO) in California. A maser was at NRAO, and at OVRO there was a Hewlett Packard 5065A Rubidium Oscillator, either running alone or driving a Sulzer 2.5-C crystal oscillator. The latter combination was strikingly better than the rubidium alone.

Cesium oscillators form the fundamental time and frequency standards, but they have traditionally been noisier than rubidium oscillators. The latest generation of cesium oscillators is said to be much improved, but they have not yet been tested in VLB systems.
Local oscillator signals are derived from the atomic oscillator outputs by various combinations of synthesizers and multipliers. These can be very stable, and contribute negligible phase noise. It must be noted, though, that some synthesizers and multipliers are very much better than others for this purpose, and the casual attachment of an LO chain to a hydrogen maser oscillator does not guarantee a stable signal.

\section{B. Long-Term Phase Stability}

A transcontinental interferometer has $d / \lambda$ ranging from $10^{6}$ to $10^{8}$, depending on wavelength, and the corresponding angular resolutions are from 0.1 to $0^{\circ} .001$. VLB systems thus have the potential for measuring source positions to very high accuracy.

The customary way to measure a position with an interferometer is to measure the phase of the interference fringes with respect to those of a standard source. In a VLB system this will fail if the relative phase between the two local oscillators drifts substantially, and unpredictably, between the two measurements. The comparison interval, then, cannot be more than the coherence time discussed in the previous section.

The simplest scheme switches the antennas back and forth between a standard source and the source under test. This has been done at $3.8 \mathrm{~cm}$ with hydrogen maser oscillators between Goldstone and Haystack. The time scale for phase stability appears to be a half-hour or more. A more complicated scheme uses two antennas at each terminal, one tracking the standard source and the other the source under test. It would be possible to record both signals all the time, but in practice they are recorded alternately, in 1-s blocks. The phase of the two sets of fringes can then be compared almost continuously, and the need for long-term phase stability is obviated. Such "four-antenna" observations have been done between NRAO and OVRO, also between NRAO and the Haystack Observatory.

A yet more complicated scheme uses three antennas at each terminal, and the redundant phase information is used to derive atmospheric corrections. One successful "sixantenna" experiment has been done between OVRO and NRAO at $21 \mathrm{~cm}$. Its object is to make high-precision measurements of the positions and motions of pulsars.

Position measurements using these techniques are discussed in detail by Counselman [18].

\section{TIMING REQUIREMENTS}

In the VLB unit [Fig. 1(b)] video signals are recorded on magnetic tapes which are processed later to find the cross correlation function. In the NRAO Mark I system, computercompatible tape is used and the processing is done on a general-purpose computer. In the Canadian system and the NRAO Mark II system, recording is on video tape. Specialpurpose processors are used to find the correlation function, which is then analyzed in a general-purpose computer.

In a conventional interferometer [Fig. 1(a)], the signals are brought into time synchronism by stepping the compensation delay line so that $\left|\tau_{i}(t)-\tau(t)\right| \ll B^{-1}$, where $B$ is the bandwidth. In the VLB unit this function is easily performed in the processor. However, the VLB interferometer lacks initial synchronization because tape starting is controlled by the two independent clocks. No attempt is made to keep these clocks synchronized to $B^{-1}$ seconds; rather, in the processor, 
many time delays are tried until fringes are found. This is occasionally a disheartening or even unouceseful process, but most recent experience has been that the tape indices are known $a$ prior $i$ to 10 or $20 \mu \mathrm{s}$, and the required amount of searching is usually small.

It has become easy to maintain time at an observatory to the accuracy required for VLB experiments. A rubidium oscillator keeps time to one part in $\mathbf{1 0}^{\mathbf{1 2}}$, or a few microseconds per month. The clocks are set against any of the network of cesium standard time stations which are maintained around the world, or by Loran-C where it is available. Even the skywave Loran signals are adequate, for they can easily give 10 or $15-\mu$ accuracy once a receiving system is calibrated.

\section{Measurement of Fringe Amplitude}

When the cross-correlation function is formed, it contains the interference fringes which form a sine wave of frequency $\nu d \tau / d t$ (to first order). This natural rate can be in the kilohertz region, and sometimes an oscillator offset is introduced for convenience, to make the net fringe rate close to zero. In most VLB experiments, only the fringe amplitude is measured, because that is easier than measuring phase and because the amplitude alone still gives most of the available information on brightness distributions. In this section we discuss measurement of fringe amplitude.

\section{A. Calibration and Signal-to-Noise Ratio}

In any interferometer system the fringe-amplitude scale must be calibrated in flux units, and this is usually done by observing standard sources, i.e., unresolved (point) sources of known strength. In VLB systems this must often be done in several steps, because there may be no sources which are known a priori to be unresolved. A second complication is that digital VLB systems use 1-b techniques and direct amplitude information is lost. Fortunately, it is easily recovered through the correlation coefficient. The 1-b correlation coefficient $\rho$ is related to the cross-correlation coefficient between the two IF signals $\rho_{12}$ by the Van Vleck formula [19] $\rho=(2 / \pi) \arcsin \rho_{12}$, and, since $\rho_{12} \ll 1$,

$$
\rho=\frac{2}{\pi} \rho_{12}
$$

$\rho_{12}$ is related to the antenna and system temperatures by

$$
\rho_{12}=\gamma\left(\frac{T_{a 1}}{T_{a 1}}\right)^{1 / 2}\left(\frac{T_{a 2}}{T_{a 2}}\right)^{1 / 2}
$$

where $\gamma$ is the unknown fringe visibility.

The optimum measurement technique consists of making careful measurement of the antenna temperature of the source $T_{a}$ and of the total system temperature $T_{a}$ for each servation. The ratios on the right-hand side of (2) are then determined. In many. cases, unfortunately, the antenna temperature cannot be measured with any accuracy because the source is weak. In these cases the usual procedure is to assume that the antenna pointing is perfect and calculate $T_{a}$ from the known total flux and the antenna gain. In principle $\gamma$ can now be determined. In practice, however, (1) is wrong by an imperfectly known number $b$, whose vahue is near 1.5 :

$$
\rho=\frac{2}{\pi b} \rho_{12}
$$

TABLE I

TELESCOpe Parameters

\begin{tabular}{lcc}
\hline Location & Diameter $(\mathrm{m})\left(\mathrm{T}_{\mathrm{s}} / \mathrm{T}_{0}\right)^{\frac{1}{2}}$ \\
\hline Arecibo, Puerto Rico & 305 & 3 \\
Goldstone, California & 64 & 6 \\
Algonquin Park, Ontario & 46 & 20 \\
Onsala, Sweden & 26 & 20 \\
\hline
\end{tabular}

because the calculation for $\rho$ uses several simplifications, such as time shifting by integral bit intervals rather than smaller values [14]. Any phase instabilities, from the oscillators or the atmosphere, also increase $b$.

The relation between $\rho$ and $\rho_{12}$ is usually unknown to 10 percent or more, and the (square-root) ratios of antenna to system temperature may also be unknown to 10 percent or more. These errors should be largely systematic and constant in any series of observations, whereas noise errors are of ten much smaller. It is customary, therefore, to attempt to eliminate the large systematic errors by scaling all values of $\rho_{12}$ by a constant factor to obtain agreement with some $a$ priori values of $\gamma$. A few sources, including PKS 0106+01, OJ 287, and $O R$ 103, have been known through experience to be smaller than others at centimeter wavelengths, and the scaling may be set to make these have unit fringe visibility. (However, this is dangerous since with sufficient resolution any source will have $\gamma<1$. Moreover, these compact sources are time varying at centimeter and decimeter wavelengths and their flux may not be known very well.) The final scaling error may still be as great as \pm 10 percent, but the relative fringe amplitudes between sources in any one series are more accurate.

With the Mark II digital system, the bandwidth $B$ is usually $2 \mathrm{MHz}$ and the typical integration time $\tau$ is $30 \mathrm{~s}$. The noise level in measuring $\rho_{12}$ can be shown to be

$$
\Delta \rho_{12} \simeq \frac{\pi b}{\sqrt{8 B \tau}} \simeq 2 \times 10^{-4}
$$

and the minimum detectable value is approximately $5 \Delta \rho_{12}$ $\simeq 10^{-3}$. To see what this means in terms of flux density, rewrite (2) as

$$
S_{c}=\gamma S=\rho_{12}\left(\frac{T_{81}}{T_{01}}\right)^{1 / 2}\left(\frac{T_{82}}{T_{02}}\right)^{1 / 2}
$$

where $S$ is the flux density of the source, $S_{c}$ is the "correlated flux density," and $T_{01}$ and $T_{02}$ are "degrees of antenna temperature per flux unit" for the two antennas. The ratio $\left(T_{0} / T_{0}\right)^{1 / 2}$ is a figure of merit, and some values for representative telescopes are in Table I. In general, this ratio is different for different wavelengths, and an optimum value is shown.

In the best cases the noise level is about $0.1 \mathrm{fu}^{1}{ }^{1}$ but for some observations it has been as high as $1 \mathrm{fu}$. Final accuracy, however, is never as good as $\pm 0.1 \mathrm{fu}$ because systematic and calibration errors dominate the error budget except in very weak cases.

Note that a low system temperature and a large effective area have equal weight in making a good figure of merit

\footnotetext{
$1 \mathrm{fu}$ stands for flux unit; $1 \mathrm{fu}=10^{-} \approx \mathrm{Wm}^{-2} \mathrm{~Hz}^{-1}$
} 
Goldstone and Onsala are relatively better than the others because they use maser amplifiers.

\section{B. Saturation}

The figure of merit $\left(T_{s} / T_{0}\right)^{1 / 2}$ is shown in Table I for weak sources, but when the source is strong enough to contribute appreciably to $T_{a}$, we must write $T_{s} / T_{a}=\left(T_{a}+T_{n}\right) / T_{a}$, where $T_{a}$ is contributed by the source and $T_{n}$ is from the receiver, the ground, etc. When $T_{a}$ is bigger than $T_{n}$, the ratio approaches unity and this side of the interferometer becomes saturated. No further improvement in sensitivity can be obtained by increasing the size of the antenna or by decreasing the receiver temperature; the signal is already due to the source itself. Saturation (defined when $T_{a}=T_{n}$ ) occurs at Arecibo at $430 \mathrm{MHz}$ when $S>10 \mathrm{fu}$, which is the case for a number of compact sources. Three sources, $3 C 84,3 C 273$, and $3 \mathrm{C} 274$, are strong enough to saturate Goldstone at centimeter wavelengths, and will also saturate the new $100-\mathrm{m}$ telescope at Bonn.

When one end of the interferometer is saturated, the system sensitivity is set by the other end. In this case the common remark that the effective area is the geometric mean is misleading. In the limit of strong saturation, (2) becomes

$$
\rho_{12} \simeq \gamma\left(T_{a 2} / T_{82}\right)^{1 / 2} .
$$

\section{BrightNess Distributions}

The simplest parameter describing the brightness distribution of a source is the effective diameter, and in many early VLB papers just this number, or an upper limit, was reported. In most cases, however, "diameter" was merely the diameter of a circular Gaussian which had the same fringe visibility as the real source, at one spacing and one position angle. Thus it was no more than a one-dimensional approximation to an "angular scale"; it of ten was meaningful and useful but also could be misleading. Better approximations to the brightness distribution are now being produced, and their sophistication is increasing.

It is well known that the output of a correlation interferometer is a component of $\Gamma(u, v)$, the Fourier transform of the brightness distribution of the source. Furthermore, the function $\Gamma$ can be synthesized by observing at different times of day and by using different spacings [20]. However, the amount of synthesis done in the typical VLB experiment is very small. At best, the source is tracked while it is above the horizon, and this gives the amplitude of the visibility function $|\Gamma(u, v)| \equiv \gamma(u, v)$ along the diurnal track, an ellipse in the $(u, v)$ plane. (Examples of diurnal tracks are in Figs. 2 and 3.) This information is grossly insufficient for any Fourier inversion, and all investigators resort to model fitting to obtain an approximation to the brightness distribution. Usually there is little $a$ priori knowledge of the source shape, and so the simplest model which fits the data is used. The simplest models contain one parameter plus a constraint on total flux; these models are circular and are usually Gaussian but can be uniform or ringlike, according to one's preferences. In most cases where the source is well resolved, there is a notable lack of circular symmetry and more complicated models must be used. The next stage is a two-parameter model, usually an equal point double with separation and position angle to be determined by the data. In a few cases this has been remarkably successful, the best and most interesting case being $3 \mathrm{C}$ 279 observed with the Goldstack interferometer at $\lambda=3.8 \mathrm{~cm}$ [16]. There is a strong attraction to double sources because it

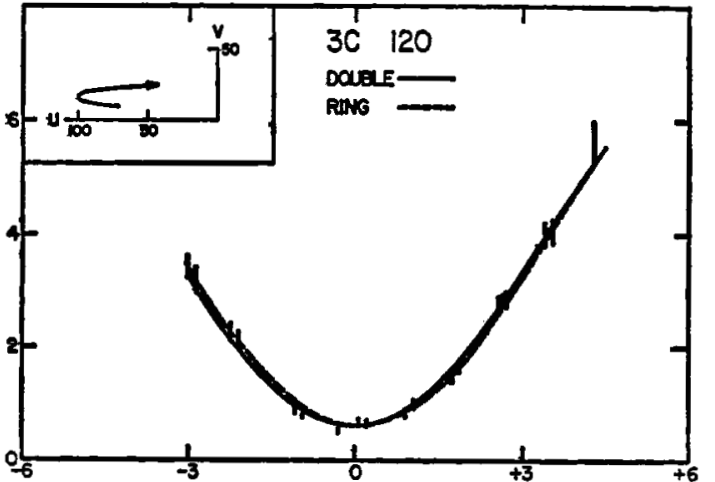

Fig. 2. Fringe amplitude data from the Goldstone-Haystack interferometer (Goldstack) at $\lambda=3.8 \mathrm{~cm}$, Feb. 28, 1971. The source is the Seyfert Galaxy 3C 120. Abscissa is the hour angle from the interferometer meridian, and ordinate is the fringe amplitude measured as the correlated flux density. The inset shows the track of the measurements on the $(u, v)$ plane, with $u$ and $v$ measured in millions of wavelengths. The two curves through the data (the vertical bars) show the expected visibility curves from two different models, a double and a ring.

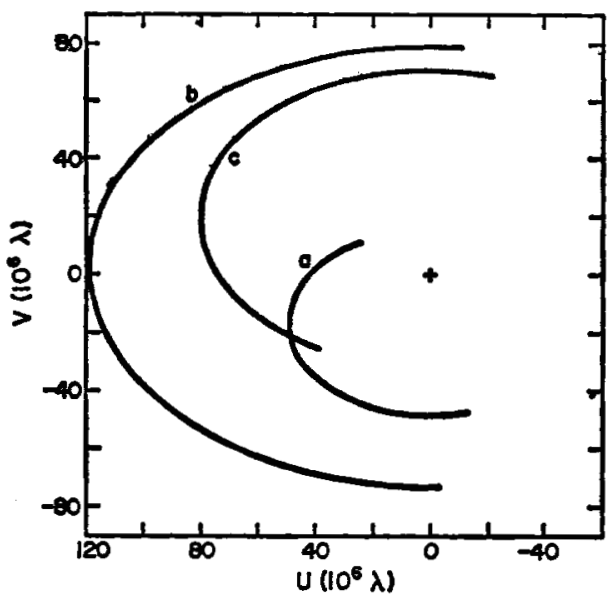

Fig. 3. Diurnal tracks on the $(u, v)$ plane for the FOG array at $2.8 \mathrm{~cm}$, $\delta=40^{\circ}$. a, OVRO-Fort Davis, $b$, OVRO-NRAO, $c$, Fort DavisNRAO.

is known that many extended radio sources are double on a large angular scale. Often the two components are symmetrically spaced across a galaxy and it is tempting to think that the galaxy has ejected the components. Can the very small doubles simply be young versions of the large mature radio sources? The answer to this is probably no, on energetic grounds, but speculation of some connection persists and colors the nature of the models which are used. (This is discussed further by Kellermann [16].)

When the equal point double does not fit the measured data, more complicated models are tried. Clearly the degree of complication can be increased until a satisfactory fit is obtained, but along the way faith in the approximation to reality may be lost, if indeed there ever was any. In many cases there are ambiguities in the model fitting. For example, Fig. 2 shows data for 3C 120 and two possible models which both fit very well, a double and a circular ring [21]. From those limited measurements it is not possible to choose between the models.

Model fitting as in Fig. 2 is inadequate to give a clear idea of the shapes of sources; in some cases no reasonable models exist and in others they are ambiguous, but in all they are unsure. To get a better view of these sources, it is necessary to sample the $(u, v)$ plane more generously. A start in this direc- 
tion is being made through several series of three-station observations. One of these is on the "FOG" array, consisting of telescopes at Fort Davis, Tex.; Owens Valley, Calif.; and Green Bank, W. Va. Fig. 3 shows tracks for the three baselines at $2.8 \mathrm{~cm}$. These are for a source at declination $+40^{\circ}$, where there are several important compact sources. The $(u, v)$ coverage for the three baselines is evidently very much better than for any one alone. Regular observations with this array are planned, to study sources which have variable brightness distributions.

One can contemplate larger arrays using existing telescopes to get more coverage in the $u,(v)$ plane and thus more angular resolution and better models. Already several four-station experiments have been done using three telescopes in the United States and one in Sweden [11]. There are more than a dozen digital Mark II recorders at observatories in North America and Europe, and one can imagine a combined assault on a few complex sources. A serious objection to this is the processing time per baseline, which is rather more than the observing time, and thus is close to half a year full-time for a two-day experiment with twelve stations. It is probable that such complicated multistation experiments will have to await the development of a satellite-linked system which could make real-time correlation of many baselines possible.

Multistation observations are also important for astrometric and geodetic measurements. If three stations are used rather than two, various closure errors can be determined, and positions found more accurately.

\section{VLB SPECTROSCOPY}

Two molecular species, $\mathrm{OH}$ and $\mathrm{H}_{2} \mathrm{O}$, show maser action in interstellar clouds and emit strong radiation in narrow spectrum lines from compact regions. The high resolution study of these lines is an active area of VLB research, and is discussed by Welch [17].

Recording on tape for line work is basically the same as for continuum work, but the processing is more complicated because, in essence, maps at many frequencies are desired, rather than just one map. In principle the analysis is made by measuring the cross-correlation function $\rho_{12}(u, v, \tau)$, including time lag. Then the Fourier transform gives the brightness distribution as a function of frequency [22]. In practice, of course, $u$ and $v$ are weakly sampled and only crude models are generated. In some cases it has turned out that a molecular source consists of a number of isolated spots of emission, each at a different frequency. A map of the overall source can then be generated even if the isolated spots have unknown shape, because each spot turns up at a different frequency and its position can be determined unambiguously [23], [24].

The $\mathrm{H}_{2} \mathrm{O}$ line is at $1.3 \mathrm{~cm}$ and the observations between Simeis, Crimea, and Westford, Mass., are the highest resolution interferometric measurements ever made, with $d / \lambda$ $=6 \times 10^{8}$. Preliminary results from these observations show that all the $\mathrm{H}_{2} \mathrm{O}$ sources are at least partially resolved [25].

In a few cases a molecular cloud produces both $\mathrm{OH}$ and $\mathrm{H}_{2} \mathrm{O}$ radiation. In these cases the diameters seem to be roughly proportional to the square of the wavelength. This suggests that the measured sizes are not intrinsic but rather are set by scattering in the interstellar medium. This conclusion remains questionable, however, because the size is an order of magnitude larger than expected on the basis of pulsar scintillations.

\section{IrRegular Propagation Effects}

Radio-wave scattering in interplanetary space and in interstellar space can have a profound influence on VLB measurements because the instrumental resolution may be comparable with the scattering angle. The phenomena are intimately connected with intensity scintillations, and the pertinent parameters will be reviewed first for them. The subject is treated in detail by Cronyn and Cohen [26].

\section{A. Intensity Fluctuations}

Three angles are important for this discussion: the scattering angle $\theta_{a} ;$ the cutoff angle $\theta_{c} \sim \lambda /\left(\theta_{s}\right)$, where $z$ is the distance to the scattering region; and the instrinsic angle of the source $\theta$. When $\theta<\theta_{c}$ we effectively have a point source, but when $\theta>\theta_{c}$ scintillations are quenched. In the first case we may imagine that the various rays within the scattering cone (as received by an antenna) are all coherent, so that they can interfere and produce the random diffraction pattern which drifts past the antenna. In the second case, however, the rays are incoherent and there is no interference. For interplanetary scintillations (IPS) $\theta_{c} \sim 0^{*} .5$, and for interstellar scintillations (ISS) $\theta_{c} \sim 10^{-6 *}$; also, for strong scattering, $\theta_{c}$ is inversely proportional to wavelength.

Scintillations are also quenched if a bandwidth limit $B_{m}$ is exceeded; $B_{m}$ is estimated from $\Delta t$, the time lag between a central ray and one from the angle $\theta_{8}$ :

$$
B_{m} \sim \Delta t^{1} \sim c /\left(z \theta_{z}^{2}\right) .
$$

This is an important limit for both IPS and ISS at low frequencies; sometimes it is called the correlation bandwidth.

The diffraction pattern on the ground has a scale $b \sim \lambda / \theta_{*}$, and if the pattern moves with velocity $u$, the time scale for fluctuations is $\tau \sim b / u$. The time scales are roughly of the order of $1 \mathrm{~s}$ for IPS, and $1000 \mathrm{~s}$ for ISS.

\section{B. Visibility Fluctuations}

The general theory of visibility fluctuations has been presented by Cronyn [27]. The main special consideration which applies to VLB work is that the antenna separation $s$ may be greater $\operatorname{than} b$, so that the antennas are in different scintillation patches. If the scintillations are strong, the amplitude and phase will vary independently in the two antennas, on the scintillation time scale $\tau$; and the integration time of the interferometer must be less than $\tau$, or else the fringes will be smeared out. Even if the scintillations in each antenna are weak, it is possible to have large phase fluctuations between the two antennas when $s \gg b$. This will happen whenever the spectrum of fluctuations in the scattering medium contains substantial energy at small wavenumbers, i.e., at scales greater than $(\lambda z)^{1 / 2}$. In this case the time limit for coherent fringes is approximately $s / u$ rather than $b / u$. Some of the attempts to measure ray bending near the sun apparently failed on this account. With the Goldstack system, $s \sim 3800 \mathrm{~km}$ and the scale for IPS is $b \sim 20 \mathrm{~km}$. The observations consisted of measuring the relative positions between two quasars as a function of solar elongation, by observing the two sources alternately and comparing the phases of the two sets of fringes. But the minimum alternation time was about $1 \mathrm{~min}$, which is larger than $s / u \sim 10 \mathrm{~s}$, and the phase comparison was meaningless. A different technique, the "four-antenna" scheme, uses two antennas at each station (as mentioned earlier); the effec- 
tive alternation time is $1 \mathrm{~s}$ and phase comparison becomes possible.

When $s<b$ the amplitude of the fringes fluctuates with the scintillation time scale, but the phase difference is steady and there is no limit to the coherent integration time.

\section{The Apparent Diameter}

It appears that all methods of measuring diameter are equivalent, and all reduce to measuring the transverse coherence in the wave field. Any conclusions derived from interferometry must also apply to measurements made in any other way, e.g., with lunar occultations, or with IPS.

There are several possible circumstances where the apparent size is the scattering angle $\theta$, rather than the intrinsic angle $\theta$. If either the integration time limit or the bandwidth limit is seriously violated, then the various rays within the scattered cone of radiation are incoherent, and the apparent size will be $\theta_{*}$. For example, VLB observations of the Crab pulsar have shown an apparent diameter of $0^{\prime} .07$ at 111 $\mathrm{MHz}$ [28]. But it is entirely implausible that this could be the intrinsic size, and so it must represent the scattering angle. For this pulsar $B_{m}$ is only about $100 \mathrm{~Hz}$ at $111 \mathrm{MHz}$, and, since the observing bandwidth was $330 \mathrm{kHz}$, the limit was seriously violated. This is an instrumental effect, for the diameter of the Crab pulsar can be measured in other ways. In particular, the observation that it shows strong ISS with a correlation bandwidth $B_{m} \sim 100 \mathrm{~Hz}$ means that $\theta<\theta_{c} \sim 10^{-7 *}$.

A more fundamental case exists when $\theta_{c}<\theta<\theta_{s}$, for now the rays within the scattering cone are intrinsically incoherent and the apparent size is $\theta$, for all measurements [26]. It is likely that many sources have size $\theta$. (interstellar) at frequencies below about $1 \mathrm{GHz}$ [29]; in fact, the only ones which do not may be the pulsars (for which $\theta<\theta_{c}$ ) and local sources such as Jupiter and spacecraft. Another conceivable exception is a molecular maser source, which may have some coherence across its face.

\section{ACKNowledgment}

The author wishes to thank W. M. Cronyn for critical remarks.

\section{REFERENCES}

[1] N. W. Broten et al., "Long baseline interferometry: A new technique," Science, vol. 156, pp. 1592-1593, June 1967.

[2] C. Bare et al., "Interferometer experiment with independent local oscillators," Science, vol. 157, pp. 189-191, July 1967.

[3] J. .M. Moran et al., "Spectral line interferometry with independent time standards at stations separated by 845 kilometers," Science, vol. 157, pp. 676-677, Aug. 1967.
[4] J. S. Gubbay and D. S. Robertson, "Nine million wavelength baseline inverferometer measurements of $3 \mathrm{C} \mathrm{273B}$," Nature, vol. 215, pp. 1157-1158, Sept. 1967.

[5] G. W. Brown, T. D. Carr, and W. F. Block, "Long-baseline interferometry of S-bursts from Jupiter," Astropkys. Let., vol. 1, pp. 89-94, 1968.

[6] B. G. Clark et al., "High-resolution observations of small-diameter radio sources at 18-centimeter wavelength," Astrophys. J., vol. 153, pp. 705-714, Sept. 1968.

[7] N. W. Broten et al., "Long baseline interferometer observations at 408 and $448 \mathrm{MHz}^{-\mathrm{I}}$. The observations," Mon. Notices Roy. Astron. Soc., vol. 146, no. 3, pp. 313-327, 1969.

[8] R. W. Clarke et al., "Long baseline interferometer observations at 408 and $448 \mathrm{MHz}$-II. The interpretation of the observations," Mon. Not. Roy. Astron. Soc., vol. 146, no. 4, pp. 381-397, 1969.

[9] M. H. Cohen, D. L. Jauncey, K. I. Kellermann, and B. G. Clark, "Radio interferometry at one-thousandth second of arc," Science, vcl. 102, pp. 88-94, Oct. 1968.

[10] M. H Cohen, "High-resolution observations of radio sources," Annu. Rev. Astron. Astrophys., vol. 7, pp. 619-664, 1969.

[11] B. F. Burke, "Long-baseline interferometry," Phys. Today, vol. 22, pp. 54-63, July 1969.

[12] K. I. Kellermann, "Intercontinental radio astronomy," Sci. Amer., vcl. 226, pp. 72-83, Feb. 1972.

[13] W. K. Klemperer, "Long-baseline radio interferometry with independent frequency standards," Proc. IEEE, vol. 60, pp. 602-609, May 1972.

[14] B. G. Clark, "The NRAO tape-recorder interferometer system," this issue, pp. 1242-1248.

[15] T. A. Clark and W. C. Erickson, "Long wavelength VLBI," this issue, pp. 1230-1233.

[16] K. I. Kelkermann, "Continuum radio sources, particularly galaxies and quasars," to be published.

[17] W. I. Welch, "OH sources and $\mathrm{H} \neq \mathrm{O}$ sources-Maser action," to be published.

[18] C. C. Counsefman, III, "Very long baseline interferometry techniques applied to problems of geodesy, geophysics, planetary science, astronomy, and general relativity," this issue, pp. 1225-1230.

[19] J. H. Van Vleck and D. Middleton, "The spectrum of clipped noise," Proc. IEEE, vol. 54, pp. 2-19, Jan. 1966.

[20] G. W. Swenson, "Synthetic-aperture radio telescopes," Annu. Rev. Astron. Astrophys., vol. 7, pp. 353-374, 1969.

[21] $\mathrm{M} . \mathrm{H}$. Cohen et al, "The small-scale structure of radio galaxies and quasistellar sources at 3.8 centimeters," Astrophys. J., vol. 170, pp. 207-217, Dec. 1971

[22] A. E. E. Rogers, "Very long baseline interferometry with large effective bandwidth for phase-delay measurements," Radio Sci., vol. 5, pp. 1239-1248, Oct. 1970.

[23] J. M. Moran et al., "The structure of the $\mathrm{OH}$ source in W3," Astrophys. J. Lett., vol. 152, pp. L97-L101, May 1968.

[24] $\mathrm{K}$. J. Johnston et al., "An interferometer map of the water-vapor sources in W49," Astrophys. J. Lett., vol. 166, pp. L21-L26, May 1971.

[25] B. F. Burke et al., "Observations of maser radio sources with an angular resolution of $0^{\prime} .0002, "$ Sov. Astron. A.J., vol. 16, pp. 379382, Nov.-Dec. 1972.

[26] W. M. Cronyn and M. H. Cohen, in preparation.

[27] W. M. Cronyn, "Interferometer visibility scintillation," Astrophys. $J$., vol. 174, pp. 181-200, May 1972.

[28] N. R. Vandenberg et al., "VLBI observations of the Crab Nebula pulsar," A strophys. J. Letl., vol. 180, pp. L27-L29, Feb. 1973.

[29] D. E. Harris, G. A. Zeissig, and R. V. Lovelace, "The minimum observable diameter of radio sources," Astron. Astrophys., vol. 8, pp. 98-104, 1970. 